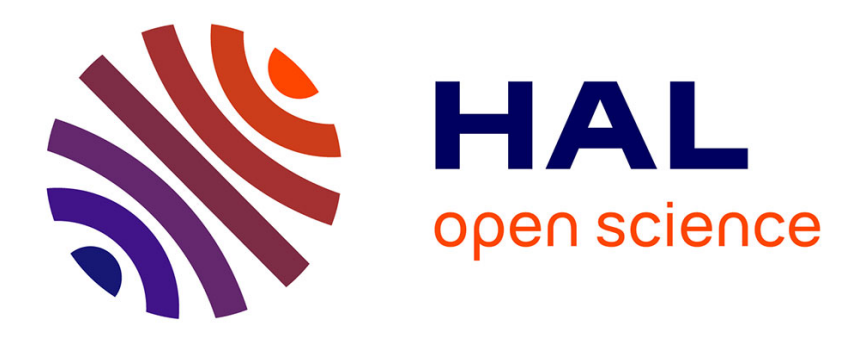

\title{
La conception et l'expression d'un droit structuré dans la société homérique à travers les notions de themis et dikê
}

Sophie Trierweiler

\section{- To cite this version:}

Sophie Trierweiler. La conception et l'expression d'un droit structuré dans la société homérique à travers les notions de themis et dikê. Archimède: archéologie et histoire ancienne, 2017, 4, pp.196206. halshs-01589419

\section{HAL Id: halshs-01589419 \\ https://shs.hal.science/halshs-01589419}

Submitted on 18 Sep 2017

HAL is a multi-disciplinary open access archive for the deposit and dissemination of scientific research documents, whether they are published or not. The documents may come from teaching and research institutions in France or abroad, or from public or private research centers.
L'archive ouverte pluridisciplinaire HAL, est destinée au dépôt et à la diffusion de documents scientifiques de niveau recherche, publiés ou non, émanant des établissements d'enseignement et de recherche français ou étrangers, des laboratoires publics ou privés. 


\section{ARCHIMÈDE}

ARCHÉOLOGIE ET HISTOIRE ANCIENNE

1 DOSSIER THÉMATIQUE 1 : NOMMER LES « ORIENTAUX » DANS L’ANTIQUITÉ

\section{DOSSIER THÉMATIQUE 2 : PRYTANÉE ET REGIA}

\section{ACTUALITÉ DE LA RECHERCHE : DYNAMIQUES HUMAINES ANCIENNES}

155 Steeve GENTNER, Thomas HUTIN et Šárka VÁLEČKOVÁ

Introduction au dossier : les phénomènes de hiérarchisation et leurs traitements dans les sociétés anciennes et actuelles

158 Pierre LE ROUX et Alain BEYRAND

D'un drame à un don. Hommage à Louise Beyrand et Olivier Toussaint

162 Alexandra CONY

Hiérarchie de I'habitat rural à la fin de l'âge du Fer. L'importance de définir les critères de hiérarchisation en fonction de l'environnement archéologique

174 Christine HUE-ARCÉ

Hiérarchies socio-professionnelles et violence interpersonnelle dans l'Égypte du Nouvel Empire et d'époque hellénistique

184 Aurélien LANDON

La hiérarchie dans le recrutement militaire : I'exemple épigraphique du recrutement des tribuns militaires durant le Principat d'Auguste

196 Sophie TRIERWEILER

La conception et l'expression d'un droit structuré dans la société homérique à travers les notions de themis et dikê

207 Francois FAVORY

Organisation et hiérarchisation de l'habitat antique : I'expérience d'Archaeomedes et d'ArchaeDyn

VARIA

216 Jean-Claude LACAM

Prestota Serfia Serfer Martier, la déesse immobile (étude ombrienne, $\mathrm{III}^{\mathrm{e}}-\mathrm{II}^{\mathrm{e}}$ siècles av. J.-C.)

229 Pierre SCHNEIDER

Des Indiens dans les armées hellénistiques : une autre rencontre entre la Grèce et I'Inde

\section{LA CHRONIQUE D’ARCHIMÈDE}

236 Frédéric COLIN (éd.)

La Chronique d'Archimède. Bilan des activités scientifiques 2016-2017 de I'unité mixte

\section{@creative @i) @(1)}




\section{LA CONCEPTION ET L'EXPRESSION D'UN DROIT STRUCTURÉ DANS LA SOCIÉTÉ HOMÉRIQUE À TRAVERS LES NOTIONS DE THEMIS ET DIKÊ}

RÉSUMÉ

Dans la société homérique, le droit s'exprime par deux notions : themis et dikê. Dans la mythologie, Themis et Dikê incarnent deux déesses apparentées, aux prérogatives bien définies. Les relations entre ces déesses précisent le sens des noms communs : de même que Dikê naît de Themis, la dikê procède de la themis, à laquelle elle doit se référer et se soumettre. Themis et dikê incarnent deux aspects du droit qui, loin de s'opposer, s'imbriquent l'un dans I'autre : themis désigne une exigence d'équilibre traduite sous la forme plus concrète de themistes, préceptes de bon comportement, dont dikê sanctionne I'observance. En d'autres termes, la dikê désigne l'attitude imposée ou la sentence formulée afin de respecter et préserver la themis, principe normatif et ordonnateur garanti par les dieux. L'analyse de ces concepts juridiques révèle ainsi l'existence d'un droit organisé dont themis incarne la finalité et dikê son application. Dans sa conception homérique, le droit dispose d'une dimension divine instaurant une seconde forme de hiérarchie parmi les acteurs du droit. Zeus apparaît comme la source d'inspiration privilégiée d'un droit dont il devient le garant et le premier représentant.

Mots-CLÉs

Autorité juridictionnelle, dikê,

droit,

normes,

hiérarchie

themis. Chargés d'appliquer le droit au nom de Zeus, les rois homériques deviennent les dépositaires d'une fonction normative et juridictionnelle déléguée par la divinité.

\author{
Sophie TRIERWEILER \\ Doctorante en histoire grecque \\ Université de Strasbourg \\ UMR 7044 Archimède \\ Sophie.Trierweiler@ac-strasbourg.fr
}

Law in the Homeric society is expressed through the notions of themis and dike. In the mythology, Themis and Dike are two related female divinities, each possessing her specific prerogatives. The relationship between the goddesses is indicative of the signification of the common names derived from them: as Dike is born from Themis, dike proceeds from themis, to which it must relate and submit. Themis and dike are two different aspects of the Greek law; far from opposing, they are intimately linked to one another. Themis refers to a need for balance, expressed through the more concrete form of the themistes; while dike is responsible for the observance of these precepts of good behaviour. In other words, dike represents the imposed human behaviour or rule expressed to respect and preserve themis, the normative and ordering principle guaranteed by the gods. The analysis of the judicial concepts of themis and dike indicates the existence of an organised and hierarchical law, whose purpose is represented by themis, whereas dike represents its application, guaranteeing harmony and social peace. The Homeric conception of law includes a divine dimension, underlining another hierarchy among the legal protagonists. Indeed, Zeus appears as the key source of inspiration of the law; he becomes its protector and first representative. Responsible for enforcing the law in the name of Zeus, the Homeric kings are then made custodians of a normative and jurisdictional function, delegated by the god.
KEYWORDS

$$
\text { dike, }
$$

hierarchy, jurisdictional authority, law, normative laws, themis. 
L'Iliade et l'Odyssée constituent les vecteurs d'un patrimoine culturel propre aux sociétés de tradition orale du haut archaïsme grec. L'absence de législation écrite n'exclut pas l'existence d'un ensemble de règles de comportement, $d$ 'interdits et d'obligations susceptible de former un système de référence à même de régir la société. Les poèmes peuvent, en ce sens, illustrer les mentalités et les pratiques juridiques des cités naissantes dominées par des membres influents de l'aristocratie locale entre le $x^{e}$ et le viII ${ }^{\mathrm{e}}$ siècle av. J.-C [1].

La société homérique est ainsi régie par un droit coutumier qui s'exprime à travers deux notions, synonymes en apparence, themis et dikê, auxquelles s'ajoutent un certain nombre de dérivés. La langue française moderne ne dispose pas d'équivalent exact pour définir ces concepts incluant notamment les idées de règle, convenance et justice. Des traductions différentes apparaissent nécessaires selon le contexte dans lequel ils sont employés. Les poèmes homériques révèlent l'existence d'un vocabulaire juridique spécifique, difficile à définir [2]

Au début $d u x^{e}$ siècle, Gustave Glotz est le premier à affirmer l'existence d'un droit structuré et distingue deux types de droit selon leur domaine d'application. Il oppose ainsi un droit familial, themis, régissant les relations internes au gênos, à un droit « interfamilial », dikê, s'appliquant aux relations entre membres de différentes tribus [3]. Toutefois, rien ne permet d'opposer concrètement ces deux notions : la themis peut s'appliquer à des situations dépassant le cadre du gênos au sens strict, tandis que les conflits qui opposent entre eux de proches parents intéressent parfois la dikê [4]. De plus, ces deux concepts peuvent être associés dans le récit d'une même action. Zeus punit « les hommes qui prononcent des themistes

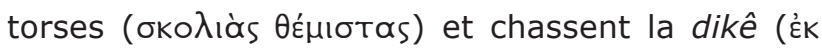

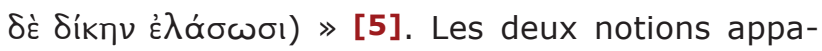
raissent suffisamment unies pour que le non-respect de I'une rejaillisse sur l'autre. Ce n'est donc pas dans l'opposition mais dans la complémentarité qu'il convient de rechercher, par une étude précise des sources, le sens de themis et dikê [6]. Ces deux notions ne se distinguent pas selon leur champ d'application, mais semblent davantage correspondre à des aspects complémentaires du droit, fondés sur la coutume et l'arbitrage.

Dans quelle mesure la transcription épique des pratiques juridiques grecques correspond-elle à la réalité du droit à la période du haut archaïsme grec ? Le droit s'exprime d'abord par deux notions étroitement liées, themis et dikê, désignant à la fois le nom d'une divinité et un concept plus abstrait. Dans ce système normatif et judiciaire dominé par la themis et la dikê, les pratiques juridiques sont encadrées par des acteurs spécifiques. Parmi eux, Zeus s'impose comme la source d'inspiration privilégiée d'un droit dont il devient le garant et le premier représentant. Chargés d'appliquer le droit au nom de Zeus, les rois homériques deviennent les dépositaires d'une fonction normative et juridictionnelle déléguée par la divinité.

\section{THEMIS ET DIKÊ : DEUX GÉNÉRATIONS SUCCESSIVES DE DIVINITÉS}

Dans l'épopée, les notions de themis et dikê désignent deux concepts abstraits, précisés par le rôle des déesses et leur lien de parenté. Les relations entre Themis et Dikê expriment dès lors la
[1] Cette étude est réalisée dans le cadre de la préparation d'une thèse de doctorat portant sur la conception du droit du haut archaïsme grec à l'époque classique, sous la direction de Cédric Brélaz. Ce travail de recherche contribue tant à l'étude de I'histoire du droit que des mentalités, de la sociologie du droit et de la place de l'écrit dans les communautés politiques.

[2] SCHeid-Tissinier 2010, p. 21-38 énumère les types d'énoncés susceptibles de véhiculer une norme et d'affirmer sa légitimité dans la société homérique. L'auteur s'intéresse à la themis mais également à un second groupe d'énoncés normatifs introduit par nemesis et lôbê.
[3] Glotz 1904, p. 19-20 repris par GERNET 2001 (1917), p. 23-24 et Benveniste 1969, p. 99-110. Plus récemment, Pelloso 2012 classe les occurrences de themis, dikê et leurs dérivés respectifs.

[4] Iliade, II, 73 ; IX, 33 ; $156 ; 297-298$.

[5] Iliade, XVI, 388. À comparer à Hésiode, Théogonie, 85-86.

[6] Les liens entre les deux notions ont notamment été étudiés par CORSANO 1988, p. 54-59 ; RUDHARDT 1999 ; Pelloso 2012. 
hiérarchie sous-tendant les concepts juridiques de themis et de dikê.

Themis est une Titanide [7], fille d'Ouranos et de Gaïa, deuxième épouse de Zeus. Cette union engendre deux générations de déesses : les Hôrai, auxquelles appartient Dikê, et les Moirai [8]. D'après la tradition hésiodique, les déesses Themis et Dikê appartiennent ainsi à deux générations successives :

« [Zeus] épousa la brillante Themis, qui enfanta les Hôrai, Eunomia (Bonne Organisation), Dikê (Justice), et Eirêné (Paix) la florissante, qui veillent sur les champs des hommes, et les Moirai auxquelles le prudent Zeus a accordé le plus grand privilège, Clothô, Lachésis et Atropos qui, seules, aux mortels donnent leur lot de bonheur et de malheur » [9].

Selon le modèle théogonique traditionnel, la descendance tend à définir et préciser les fonctions du géniteur [10]. Conformément au processus de différenciation, de spécification et d'individualisation croissante au rythme des générations divines, les qualités et les fonctions de la descendance de Themis explicitent les caractéristiques concentrées par la déesse. Or, par le champ d'action qui est le leur, les filles de Themis ont vocation à intervenir dans la vie politique et juridique de la communauté [11]. Chacune d'elles incarne un principe essentiel au maintien de l'équilibre : les notions de Bonne Organisation, de Justice et de Paix tendent à conférer à Themis une fonction d'ordonnance sociale [12]. En s'unissant à Themis, Zeus fait sien I'ordre dont la déesse est porteuse et l'associe ainsi à l'établissement de sa nouvelle souveraineté [13]. Themis est donc non seulement associée au nom de Zeus, mais également à la souveraineté qu'il

[7] Hésiode, Théogonie, 135.

[8] Pironti 2009, p. 13-27 analyse la relation entre la sphère de la themis et les Moirai, puissances de distribution, de rétribution et de régulation au service de Zeus.

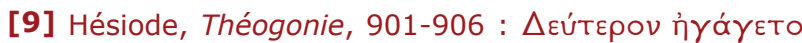

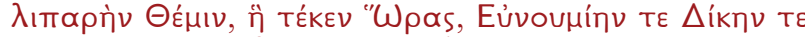

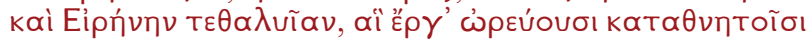
ßротоїбı, Moípas 日' (...).

[10] Selon RUdHARDT 1999, p. 44, les Titans constituent des divinités peu individualisées dont la « principale fonction fut d'engendrer des enfants mieux spécifiés qu'eux, dans lesquels les qualités que chacun d'eux portait s'explicitent et se définissent. [...] ils remplissent avec précision les fonctions que leurs parents exerçaient confusément ».

[11] RUDHARDT 1999, p. 151-154 illustre l'action conjointe des Hôrai pour la préservation de l'ordre social. Cf. également : Hésiode, Travaux et Jours, 225-229.

[12] Lo Schiavo 1997 analyse les rapports entre Thémis et la notion d'ordre cosmique. incarne. L'Hymne à Zeus chante «le plus puissant et le plus grand des dieux [...], celui qui s'entretient souvent avec Themis siégeant à ses côtés »[14]. La déesse fait office de conseillère privilégiée du souverain olympien [15].

Dans l'épopée, Themis est étroitement liée à la vie politique et délibérative [16]. Déesse de la justice et de l'équité, elle est celle qui mobilise et dissout les assemblées. Dans I'Iliade, Zeus ordonne à Themis « de convoquer les dieux à l'assemblée. Elle va donc de tous côtés leur porter l'ordre de se rendre au palais de Zeus » [17]. De même, chargée de transmettre les directives du Cronide, Héra rejoint I'Olympe et «trouve les autres dieux immortels assemblés dans le palais de Zeus ». Elle est accueillie par Themis « courant au-devant d'elle », puis lui prescrit « d'ouvrir le banquet » [18] au cours duquel les dieux ont coutume de délibérer. Dans l'Odyssée, alors que, dans la demeure d'Ulysse, les prétendants enfreignent les règles de I'hospitalité depuis près de dix ans, Athéna suggère à Télémaque de convoquer l'assemblée d'Ithaque pour témoigner devant tous de la situation dont il est victime et d'exiger publiquement le départ des prétendants. À cette fin, il invoque Zeus Olympien et « Themis qui convoque ou dissout les assemblées du peuple »[19]. Puissance de rassemblement et d'ordonnancement des communautés, la déesse semble donc jouer un rôle essentiel dans la prise de décisions lorsque les dieux ou les hommes se réunissent pour délibérer.

En revanche, Dikê, une des trois Hôrai issues de Zeus et Themis, n'est jamais mentionnée dans l'épopée homérique ; elle fait toutefois l'objet d'un grand intérêt chez Hésiode.

[13] Le mythe fait de Thémis l'une des détentrices de I'oracle de Delphes. Ce pouvoir prophétique lui confère un rôle décisif dans le destin de Zeus. En I'informant de la menace qui pèse sur la longévité de son règne, Thémis se pose comme garante de sa souveraineté et de l'ordre établi, renforçant ainsi le lien qui l'unit à Zeus. Cf. notamment Pindare, Isthmiques, 8, 22-49.

[14] Hymne homérique à Zeus, 1-3.

[15] Sur le culte rendu à Thémis, cf. RUDHARDT 1999, p. 43-57. L'invocation conjointe de Zeus et Thémis perdure dans la tradition littéraire postérieure. Cf. notamment Euripide, Médée, 169 ; 208 ; 1160 ; Sophocle, Électre, 1063-1064 ; Eschyle, Les Suppliantes, 359-360. Thémis fait souvent office de parèdre de Zeus.

[16] Du SABLON 1999, p. 188-195 analyse les trois passages de la Théogonie consacrés à Thémis. Hésiode insiste sur le principe organisateur de Thémis au détriment de l'aspect rassembleur illustré dans l'épopée.

[17] Iliade, XX, 4-6.

[18] Iliade, $\mathrm{XV}, 87-95$.

[19] Odyssée, II, 69. 
Dikê appartient à ces «trente milliers d'Immortels [qui], sur la glèbe nourricière, sont, de par Zeus, les surveillants des mortels, et surveillent leurs sentences, leurs œuvres méchantes, vêtus de brume, visitant toute la terre ». Interpellant les rois, Hésiode les exhorte à respecter la justice et à « [songer] aussi qu'il existe une vierge, Dikê, la fille de Zeus, qu'honorent et vénèrent les dieux, habitants de I'Olympe. Quelqu'un I'offense-t-il par de tortueuses insultes ? Aussitôt elle va s'asseoir aux pieds du Cronide, son père, et lui dénonce le cœur des hommes injustes » [20].

À l'instar de Themis, Dikê est directement associée au nom de Zeus et à sa souveraineté. Elle revêt le rôle de collaboratrice privilégiée qui siège aux côtés de son père [21] et l'assiste dans ses fonctions. Chargée de surveiller le comportement des hommes, elle lui désigne ceux qui, parmi eux, agissent de manière injuste [22]. La récompense de I'homme juste et le châtiment de I'homme injuste sont présentés comme le strict résultat de la volonté et de l'action de Zeus [23]. Si chez Hésiode, Dikê a davantage un rôle d'observation, laissant à Zeus la tâche de punir les coupables, elle n'en reste pas moins fortement impliquée dans la répartition des peines et des récompenses. Elle apparaît alors telle une puissance distributrice chargée d'assurer et de sanctionner le respect des lots attribués à chacun afin de garantir l'équilibre incarné par Themis [24]. Assistant volontiers les hommes justes, elle les accompagne et les soutient dans leurs projets tandis qu'elle ignore les hommes qui, attirés par I'hybris, se livrent à des comportements répréhensibles. Polynice arbore un bouclier sur lequel figure la déesse Dikê accompagnée d'une légende en lettres d'or témoignant du soutien que lui procure la déesse dans sa lutte vengeresse [25]. Étéocle, quant à lui, blâme le comportement de son frère et ne peut concevoir que la déesse l'accompagne, elle qui « jamais encore, $[\ldots]$ ne l'a honoré d'un mot; et ce n'est pas au moment où il meurtrit la terre de ses pères qu'elle peut être à ses côtés ou elle serait alors entièrement infidèle

[20] Hésiode, Travaux et Jours, 256-261.

[21] Cf. Sophocle, Erdipe à Colone, 1382 où Dikê partage le trône de Zeus.

[22] L'approche d'Hésiode se retrouve dans un papyrus $\mathrm{d}^{\prime} \mathrm{Oxy}$ rhynque dont les vers sont attribués à Eschyle, ( $P$.Oxy. XX 2256 fr. 9a) cité dans RUDHARDT 1999, p. 144. Interpellée par le chœur, Dikê s'exclame : « Je suis assise sur le trône de Zeus, entourée de gloire. Il m'envoie lui-même auprès de ceux pour lesquels il a de la bienveillance, Zeus, lui qui m'a, dans de bonnes intentions, envoyée sur la terre. [...] [On me à son nom, cette Dikê qui s'associerait à un homme dont l'audace ne recule devant rien » [26].

Dikê remplit donc la fonction d'agent messager dont les observations provoquent des sanctions administrées directement par le maître de l'Olympe ou en son nom par Dikê qui influence les actions des hommes. Themis et Dikê incarnent différents aspects du droit, chacun étroitement lié au nom de Zeus, souverain par excellence.

La mythologie apporte une première indication sur le rôle de Themis et de sa fille Dikê. Les deux déesses sont directement associées au nom de Zeus : I'une par le mariage, I'autre par son ascendance paternelle. Leurs fonctions respectives se trouvent dès lors étroitement liées à la souveraineté : Themis et Dikê sont solidaires de l'équilibre garanti par l'autorité souveraine de Zeus, conférant ainsi à la divinité un rôle déterminant dans la conception du droit, comme source d'inspiration des normes coutumières et garante de leur application.

La dimension normative de Themis et Dikê s'exprime ensuite à travers leurs attributions respectives. Loin de signifier une simple accumulation de prérogatives, la polyvalence des divinités suppose un réseau articulé de notions dont les concepts de themis et dikê se font l'écho. Themis concentre les trois notions de justice (Dikê), de bonne organisation ou d'harmonie (Eunomia) et de paix (Eirêné). Cette imbrication symbolisée par les généalogies successives se retrouve dans le lien qui unit la themis à la dikê, la seconde devant respecter la première. Tout comme Dikê naît de Themis, la dikê procède de la themis.

\section{THEMIS ET DIKÊ : DES CONCEPTS ABSTRAITS DÉFINISSANT UN DROIT STRUCTURÉ FONDÉ SUR LA COUTUME ET L'ARBITRAGE}

Le droit homérique s'exprime à travers les notions de themis et dikê, dont la complémentarité et l'influence réciproque sont suggérées par le rôle et la nomme] Dikè [...]. Aux justes je donne en récompense une vie justement accordée à leur vertu. Quant aux sots orgueilleux, je fais grandir en eux un esprit sage [...] en inscrivant leurs fautes sur les tablettes de Zeus ».

[23] Hésiode, Travaux et Jours, 238-240.

[24] En ce sens, les Moirai, comme puissances de répartition et d'accomplissement, peuvent faire écho à cet aspect de Dikè. Cf. Pironti 2009, p.16-20.

[25] Eschyle, Sept contre Thèbes, 642-649.

[26] Eschyle, Sept contre Thèbes, 658-671. 
parenté des déesses. L'étymologie et l'analyse des occurrences permettent de préciser le sens de ces notions et révèlent l'existence d'un droit hiérarchisé dès l'époque homérique [27].

\section{LES EMPLOIS DE THEMIS}

Le terme themis se fonde sur la racine indo-européenne *dhê- signifiant « poser d'une manière créatrice, placer, établir dans l'existence », à rapprocher de l'avestique dà-mi « création » et du sanscrit dhaman «loi », « siège », désignant à la fois ce qui est établi et le lieu de son établissement. Sur cette même racine se forment le verbe latin facio et le verbe grec tithêmi « poser, placer, établir » dont dérive thesmos « ce qui est établi, règle, norme » [28].

L'étymologie précise ainsi l'aspect normatif véhiculé par themis et évoque sans ambiguïté son sens institutionnel. En tant que nom commun, themis, revêt deux significations sensiblement différentes selon qu'il est employé au singulier ou au pluriel, themistes.

Au singulier, themis apparaît presque toujours

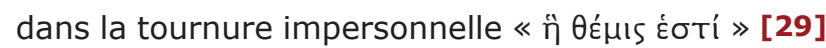
littéralement «ce qui est themis », ou ses variantes négatives [30], et désigne «ce qui est établi par la coutume, conforme à l'usage » [31]. Sous cette forme, il peut s'agir d'une loi naturelle [32], d'une règle morale [33], d'un simple usage, d'une norme sociale ou religieuse.

La formule homérique désigne ce qui semble n'être de prime abord qu'une simple habitude. Achille mentionne par exemple les Achéens membres du Conseil « qui, chez [lui], sans cesse ( aỉ̇i) entrent s'asseoir et

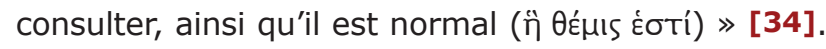
L'utilisation de l'adverbe aiei traduit le caractère répétitif de la scène. Souvent, ces habitudes tendent à devenir de véritables règles de comportement. L'usage devient alors une conduite imposée par les

[27] Pelloso 2012, p. 153-174 énumère les occurrences de themis, dikê et leurs dérivés dans I'Iliade et l'Odyssée. [28] BenVeniste 1969, II, p. 12.

[29] Iliade, II, 73 ; IX, 33 ; IX, 134 ; XI, 779 ; XIX, 177; XXIII, 58.

[30] Iliade, XIV, 386 ; XVI, 79 ; XXIII, 44.

[31] Chantraine 2009, Dictionnaire étymologique de la langue grec, s.v. $\theta \dot{\varepsilon} \mu l s$.

[32] Iliade, IX, 134 ; IX, 276 ; XIX, 177 ; Odyssée, XIV, 130.

[33] Odyssée, XI, 451.

[34] Iliade, XXIV, 652.

[35] Odyssée, XI, 451

[36] Odyssée, XXIV, 286. Concernant les règles de l'hospitalité, cf. notamment Odyssée, III, 187 ; IX, 268 ; X, 73. convenances et la tradition. Lorsque la themis se réfère au respect des règles de I'hospitalité et du culte des morts, elle renvoie à une norme sociale primordiale dans la société héroïque. Échoué sur lî̂le du Cyclope Polyphème, Ulysse réclame que celui-ci « [leurs] donne [à lui et ses compagnons], selon l'usage

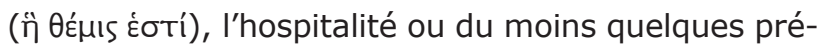
sents » [35]. Le code de I'hospitalité implique par ailleurs une sorte d'équité dans la réciprocité [36]. Appliquée au domaine du rituel, themis concerne les règles relatives à la pureté et à l'impureté, au culte des morts ainsi qu'aux conduites consécutives à un meurtre [37]. Enfin, la themis est liée à la procédure et au respect du serment [38].

La themis apparaît comme une notion polysémique difficile à définir et suffisamment générale pour concerner un large champ de domaines. La pluralité de ses emplois nécessite de recourir à des traductions différentes car la langue française ignore cette notion incluant notamment normalité, justice et convenance [39]. Toutefois, son sens profond reste le même. La themis s'exprime par les comportements et les attitudes qu'elle prescrit ou qu'elle proscrit. D'un point de vue juridique, est qualifié de coutume tout usage suffisamment répandu dans l'espace et dans le temps, tout usage à caractère ancien régulièrement suivi par une communauté. Le héros qui se réfère à la themis, se réfère donc à un usage coutumier et inscrit son geste ou sa parole dans une tradition qui se fait norme. Dans l'épopée, la themis ne désigne donc pas une règle impérative sûrement établie et clairement énoncée mais une habitude qui par sa répétition devient norme sociale, autrement dit, un usage consacré par la tradition qui devient coutume, prenant ainsi force de loi.

Une occurrence fait toutefois figure d'exception et permet d'affiner le sens de themis. Dans I'Iliade,
[37] Odyssée, III, 45 ; Iliade, XVI, 796 ; XXIII, 44. Cf. également Odyssée, XIV, 56 et pour des occurrences plus tardives de themis relatives au culte funéraire et au respect des règles rituelles, cf. notamment Sophocle, Fipe à Colone, 1188-1191.

[38] Iliade, XXIII, 581. Cf. également Euripide, Médée, 208 où le chœur invoque « Thémis, la fille de Zeus, gar-

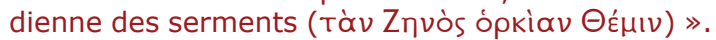

[39] Les traductions de Paul Mazon évoquent cette perception. Pour lui, « il est themis » renvoie tantôt à l'idée d'une attitude normale inscrite dans l'ordre naturel des choses « c'est normal, ce n'est pas normal », tantôt à I'idée d'équité « c'est justice (Odyssée III, 187) 》; «ce n'est pas l'équité (Odyssée, XXIV, 286) 》 ou encore à l'idée d'un comportement autorisé ou non, d'un usage inscrit dans les consciences collectives qu'il est de règle de respecter « il est admis (Iliade XVI, 796) 》; « il n'est pas admissible ; il n'est pas permis (Odyssée $X, 731) »$. 
Patrocle longe les nefs d'Ulysse « où se tiennent I'agora et la themis et où ont été élevés les autels

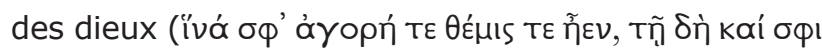

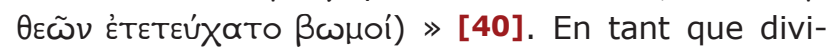
nité, Themis, déesse des assemblées, incarne parfaitement cette complémentarité. Chez les hommes, elle garantit les règles de la délibération. En I'occurrence, les nefs d'Ulysse se situent au centre du camp achéen. Le couple agora/themis désigne le lieu de rassemblement par excellence, symbole de cohésion et d'unité, autrement dit le lieu de la délibération et de la décision politiques. Cette proximité permet précisément de souligner la dimension politique de themis, liée au respect des règles de vie en société. Les décisions prises sur l'agora respectent la themis. L'homme qui aime la guerre civile est un être « sans

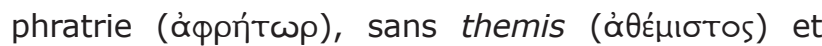
sans foyer (ávéotıos) » [41], incapable de construire des liens stables à même d'assurer toute intégration dans la société. La themis incarne donc ce principe organisateur des communautés, fondamental pour mener une vie civilisée [42].

Dans sa dimension normative et politique, la themis désigne l'exigence d'équilibre, le principe qui confère à la coutume sa valeur normative afin de préserver I'ordonnance sociale.

\section{LES EMPLOIS DE DIKÊE}

La seconde notion juridique présente dans l'épopée est celle de dikê. Contrairement à la themis, l'étymologie n'explique pas immédiatement la dimension normative ou judiciaire véhiculée par dikê. Celle-ci recouvre deux significations différentes issues de la racine indo-européenne *deik, de laquelle dérivent le latin dico « dire » et le grec deiknumi « montrer ». Apparemment indépendants, ces deux sens semblent s'être développés en parallèle [43]. Dikê désignerait d'une part « le signe, la marque, la caractéristique », et d'autre part «la limite, la ligne de démarcation », à partir de laquelle s'est développé le sens de jugement ou de sentence, destiné métaphoriquement à instaurer une limite, une séparation entre deux parties en conflit.

Dans l'épopée, le premier sens s'exprime par la

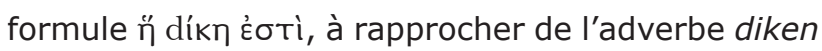
« à la manière de ». Sous cette forme, dikê apparaît dans sept passages de l'Odyssée et désigne la manière d'être ou d'agir, le comportement traditionnel, propre à une catégorie [44]. Dans les six premiers exemples, dikê est directement suivi d'un génitif pluriel qui indique le groupe caractérisé. Dans le dernier, elle précède une subordonnée temporelle qui présente néanmoins le même caractère général employé pour singulariser un groupe d'individus [45]. Ainsi, la dikê des rois (n̋ T'żotì

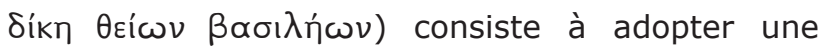
attitude exemplaire sans jamais rien dire ou faire pour abuser leur peuple [46], celle des morts ( $\alpha \lambda \lambda$ '

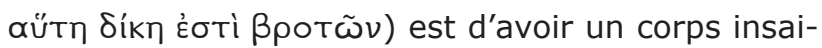

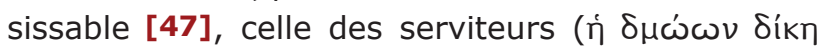
Éotiv) de craindre leurs jeunes maîtres [48], celle

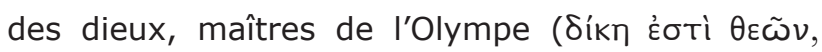
oi "O se manifestant [49] et celle des vieillards (í Síkn

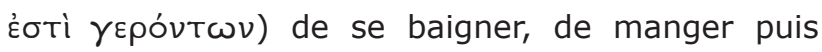
de dormir [50]. Pénélope déplore les mauvaises manières des prétendants dont le propre, la dikê, n'est pas de s'approprier les biens de celle qu'ils

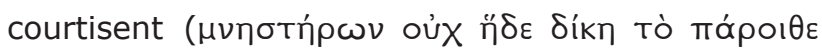
тย́тUкто), mais au contraire de lutter de générosité pour la conquérir [51].

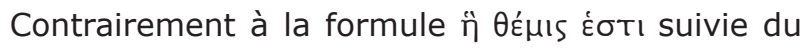
datif, il ne s'agit pas d'appliquer une règle générale à un cas particulier, mais de reconnaître la singularité d'un groupe d'individus. Ainsi, alors que la themis semble universelle, la dikê se définit d'emblée par
[40] Iliade, XI, 807-808. Sur ce passage, cf. RUDHARDT 1999, p. 28 ; Ruzé 1997, p. 41-44 et BouvieR 2002, p. $262-264$.

[41] Iliade, IX, 63-64; à rapprocher de Iliade, V, 759-811.

[42] Odyssée, IX, 112 : les Cyclopes ne possèdent « ni assemblées délibérantes ni themistes (oüré áyopaì

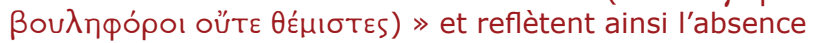
de toute organisation sociale.

[43] Palmer 1950 , p. $149-168$ suivi par Gagarin 1973 , p. $81-94$ et RUDHARDT 1999, p. 104-106 ; contra BENVENISTE 1969, p. 111, qui se réfère à la même racine mais n'évoque qu'un seul sens, restant ainsi tributaire de Glotz 1904 et HiRzel 1907 p. 104-108. Pour un état de la question, cf. Du SABLON 2014, p. 241-263.

[44] RUDHARDT 1999, p. 111-112 propose de percevoir dans cette formule «le lot convenant à un individu » ou à un groupe d'individus, « ce qui revient de droit à un individu, sa part, son droit ».

[45] Odyssée, XIX, 167-168.

[46] Odyssée, IV, 690-691.

[47] Odyssée, XI, 218-222 : « Pour tous, quand la mort nous prend, voici la loi » (éd. Bérard). La traduction par « loi » proposée par Victor Bérard traduit la dimension normative reconnue à une situation conçue comme inévitable et systématique, définissant une règle générale unanimement admise.

[48] Odyssée, XIV, 59-61.

[49] Odyssée, XIX, 43.

[50] Odyssée, XXIV, 254-255.

[51] Odyssée, XVIII, 275-278. 
la considération de qualités propres aux individus qu'elle concerne. En définissant ainsi le type de comportement qui caractérise telle ou telle catégorie d'individus, ce type d'énoncé peut servir à véhiculer une norme coutumière. Plus qu'une manière d'être ou d'agir, la formule ñ díkn k̇otì tend à désigner une règle impérative propre à un groupe ou une catégorie sociale. Par sa répétition, le comportement habituel devient comportement attendu. Il s'inscrit dans une continuité, s'ancre dans une tradition socialement consacrée et acquiert ainsi une dimension normative.

Avant de prendre le sens moral de justice et d'incarner le principe organisateur de la société dans la Grèce classique, dikê appartenait au vocabulaire judiciaire [52]. Dans sa seconde acception, dikê désigne le jugement prononcé ou la sentence, destiné métaphoriquement à instaurer une limite, une séparation entre deux parties en conflit [53].

Dans l'épopée, quiconque a le sentiment que sa timê [54], son honneur, est remis en cause, peut recourir à la force pour obtenir réparation ou engager une procédure judiciaire. Dans le premier cas, le recours s'identifie à la vengeance, dans le second, il s'agit d'un recours amiable dont la forme privilégiée est l'arbitrage. Les plaideurs peuvent recourir à un arbitre reconnu comme tel par la com-

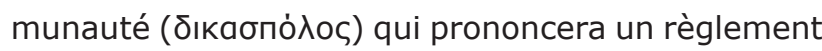

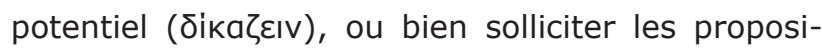
tions de différentes figures d'autorité. Au cours de cette procédure, les plaideurs peuvent eux-mêmes proposer un arbitrage [55]. En l'absence d'institution judiciaire permanente, la dikê apparaît comme la solution ponctuelle de différends privés.

Dans la scène judiciaire figurée sur le bouclier d'Achille [56], deux hommes se disputent le prix du sang (poinê) et soumettent leur cas aux anciens siégeant sur l'agora, devant la foule venue assister

[52] Gagarin 1973, p. 81-94 privilégie cette dimension qu'il considère comme fondamentale dans la pensée archaïque contra DICKIE 1978, p. 91-101 qui confère une dimension morale à la dikê dès le haut archaïsme.

[53] Benveniste 1968, p. 109, explique la transition entre la notion de montrer d'une part et celle de dire d'autre part puis associe la racine $*$ deik à l'idée de montrer verbalement. Il se réfère ensuite à l'expression latine dicis causa et au concept sous-jacent de justice, pour définir dikê comme « le fait de montrer avec autorité de parole ce qui doit être ».

[54] Le respect de la timê d'autrui constitue une règle fondamentale des rapports sociaux dans la société aristocratique décrite par les poèmes homériques. Pour une étude de la timê comme valeur structurante des sociétés homériques, cf. Du SABLON 2014, p. 23-56.

[55] Iliade, XXIII, 542-544. à la scène. L'audition des plaideurs achevée, les anciens se lèvent et, sceptre en main, prononcent

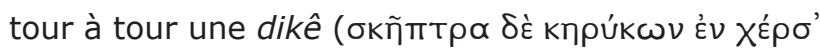

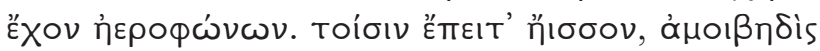
$\delta \varepsilon \dot{~} \delta i ́ k \alpha \zeta o v)$. Associé à themis et dikê, le sceptre incarne la procédure réglementée : toute sentence est prononcée sous couvert du sceptre. Au milieu des anciens se trouvent deux talents d'or destinés à « celui qui, parmi eux, énoncera l'avis le plus

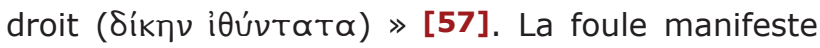
collectivement son approbation ou son désaccord et contribue à l'élaboration d'une sentence à même de restaurer la concorde et d'apaiser durablement la communauté. Spectatrice active, la foule exerce une influence, voire un contrôle, sur la décision. À mesure que les orateurs se succèdent et développent leur propre sentence, une décision prend forme et tend à s'imposer. La dikê la plus droite ( $\delta i ́ k \eta v$

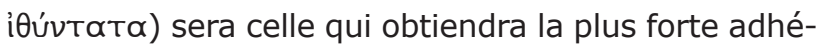
sion. La composante publique de la dikê garantit une sentence juste, sanctionnée par la communauté.

La dikê semble ainsi désigner la sentence ou le règlement public, prononcé solennellement par le juge, ou plus rarement par le plaideur, qui doit amener une solution pacifique au différend en faisant droit aux parties [58]. Par extension, dikê peut également désigner la procédure elle-même ou le contenu de l'arbitrage approuvé par les plaideurs [59]. Elle désigne alors la compensation réparatrice que la victime est en droit de recevoir au regard de sa timê pour que soit restauré l'équilibre (themis) qui a été dans un premier temps compromis à ses dépens.

La dikê est liée au respect des règles de vie en société. Toutefois, la pression sociale qui tend à rendre des dikai justes n'est possible que parce qu'il existe des normes communes reconnues par tous : les themistes, conçues comme des prescriptions de la themis.

[56] Pour un état de la question, cf. CANTARella 2002, p. 147-165.

[57] Iliade, XVIII, 497-508.

[58] LÉvy 1998, p. 73, dans le respect de la timê. SEALEY 1994, définit la dikê comme le mode de preuve utilisé pour résoudre un différend. Cependant, une telle définition ne concorde pas avec la finalité même du droit homérique fondé sur une justice processuelle administrée au cas par cas dont la finalité repose sur l'adoption d'un arbitrage unanimement consenti par les plaideurs.

[59] Iliade, XIX, 180. À l'occasion de la cérémonie publique qui sanctionne la réconciliation entre Achille et Agamemnon, Ulysse énumère les démarches qu'Agamemnon doit accomplir pour satisfaire I'honneur blessé du héros, afin que « rien ne [lui] manque de ce qu'exi-

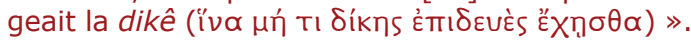


Dépourvus de themis et de dikê, les Cyclopes « ignorent toute possibilité de régler pacifiquement

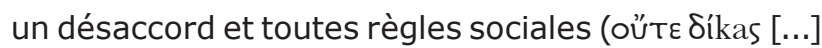

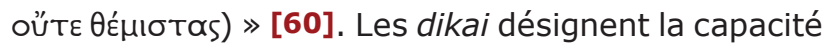
d'établir un compromis équitable pour les parties. Or, sans themistes, les Cyclopes sont incapables de constituer une communauté sociale organisée et, à I'instar de Polyphème, sont réduits à dicter et imposer leurs décisions par la force. Par ailleurs, l'emploi de themistes pour fonder les jugements

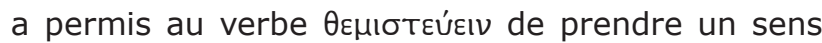
judiciaire et non plus strictement juridique [61]. Il se distingue de $\delta ı$ á̧́દıv qui n'apporte qu'une solution ponctuelle en se fondant sur des règles antérieures

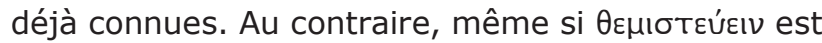
employé pour un cas particulier, la décision rendue aura valeur générale et sera à même de constituer un précédent. Les themistes désignent donc non seulement les règles traditionnelles reconnues de tous qui fondent les jugements (dikai) mais également les sentences qui fonderont les jugements futurs et feront jurisprudence. Elles ont donc un caractère général que les dikai n'ont pas [62]. Entre themis et dikê, il existe donc une hiérarchie exprimée par le caractère général de l'une auquel l'autre, plus spécifique, doit se soumettre. De même que Dikê naît de Themis, la dikê procède de la themis, à laquelle elle doit se référer et se conformer. Achille exprime parfaitement ce lien quand il s'adresse aux

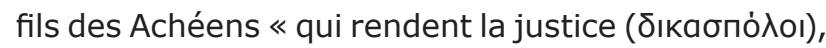
et au nom de Zeus, maintiennent les themistes (oí тع

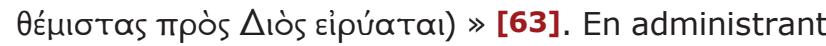
convenablement la dikê, les juges maintiennent l'équilibre incarné par la themis.

Themis et dikê représentent deux aspects du droit qui, loin de s'opposer, s'imbriquent l'un dans l'autre : themis désigne une exigence d'équilibre traduite sous la forme plus concrète de themistes, préceptes de bon comportement, dont dikê sanctionne I'observance. En d'autres termes, la dikê désigne l'attitude imposée ou la sentence formulée afin de respecter et préserver la themis, principe normatif et ordonnateur garanti par les dieux.

[60] Odyssée, IX, 215.

[61] Odyssée, XI, 569-571 : Minos, « ce roi siégeait pour

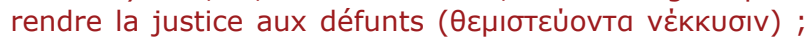
assis autour de lui ou debout, les plaideurs emplissaient la maison d'Hadès ».

[62] BenVeniste 1968, p. 99-110, conçoit les dikai comme des formules de droit qui se transmettent et que le juge a charge de conserver et d'appliquer. Toutefois, les dikai, comme règlement prononcé par une autorité juridictionnelle destiné à mettre fin à un conflit sans user
L'analyse de ces concepts juridiques révèle ainsi l'existence d'un droit organisé dont themis incarne la finalité, et dikê son application.

Dans sa conception homérique, le droit dispose d'une dimension divine instaurant une seconde forme de hiérarchie parmi les acteurs du droit chargés de préserver la themis en respectant la dikê.

\section{UN DROIT COUTUMIER ENCADRÉ PAR LA DIVINITÉ}

La société homérique est régie par un droit coutumier encadré par la divinité. Zeus apparaît comme la source d'inspiration privilégiée d'un droit dont il devient le garant et le premier représentant.

La themis et la dikê apparaissent comme des dons de Zeus. Hésiode présente la dikê comme une création de Zeus et affirme ainsi : «tel est l'usage (vónov) que le Cronide a prescrit aux hommes : que les poissons, les fauves, les oiseaux ailés se dévorent, puisqu'il n'est point de dikê parmi eux

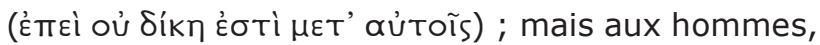
Zeus a fait don de la dikê, qui est de loin le premier

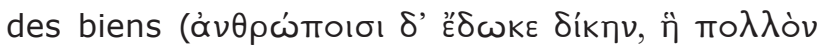

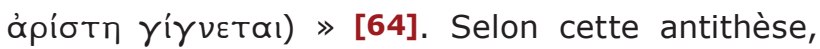
quiconque ne se conduit pas avec dikê n'est autre qu'un animal, une brute incivilisée à l'instar des Cyclopes. Se conduire avec dikê consiste à adopter un comportement conforme aux themistes ou à rendre un arbitrage à même de préserver la themis. Dans l'épopée, l'exercice de la justice est associé au dikaspolos, littéralement « celui qui rend les dikai » [65]. À défaut de substantif pour désigner le juge homérique, l'anêr dikaspolos qualifie l'homme chargé d'exercer la justice par voie d'arbitrage, autrement dit, l'individu socialement habilité à exercer I'autorité juridictionnelle. Dans I'Odyssée, Ulysse interroge le fantôme de sa mère Anticléia sur le sort de son père et de son fils. Pour le rassurer, Anticléia affirme que Télémaque « exploite en paix la terre royale et prend sa juste part aux festins coutumiers comme il sied à qui administre

de la violence, ont une valeur purement ponctuelle et n'ont pas vocation à faire jurisprudence.

[63] Iliade, I, 238-239.

[64] Hésiode, Travaux et Jours, 278-279.

[65] BeNVENISTE 1968, p. 109 propose « celui qui veille sur les dikai » mais cette définition connote à tort l'idée d'un recueil de sentences sur lequel le juge s'appuierait pour rendre la justice, et contreviendrait à la conception homérique du droit prônant la résolution des différends au cas par cas. 


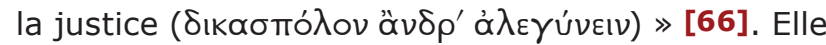
dresse l'image d'un fils qui détient toujours l'usufruit des biens royaux et assume les devoirs de son père [67], laissant ainsi largement supposer que la fonction juridictionnelle attribuée à Télémaque représente une fonction habituellement dévolue aux rois. L'adjectif dikaspolos désignerait donc la fonction juridictionnelle du roi homérique. Dans I'Iliade, Achille qualifie de dikaspoloi « ceux qui rendent la

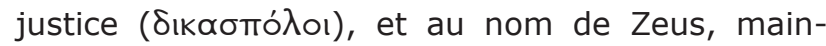

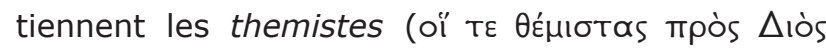
Eipúataı) » [68]. La forme plurielle dikaspoloi laisse envisager qu'il pourrait s'agir du groupe des anciens dont la fonction juridictionnelle est évoquée dans la scène judiciaire gravée sur le bouclier d'Achille [69]. $\mathrm{Au}$ regard de leurs attributions, les anciens sont également des rois homériques [70]. Les acteurs du droit incarnent différents stades dans I'histoire de l'administration de la justice. Les poèmes évoquent ainsi conjointement la tradition d'une justice mycénienne administrée par un wanax aux pouvoirs puissants et indifférenciés, incarné par Minos, et la conception judiciaire propre aux cités naissantes où les basileis, rois homériques, interviennent de manière individuelle ou collégiale dans le règlement des conflits pour rétablir l'équilibre et préserver I'ordre social [71].

Or c'est précisément aux rois que Zeus octroie les themistes [72]. Ulysse rappelle ainsi que Zeus « donne le sceptre et les themistes au roi afin que pour eux [les Achéens] il décide » [73]. Autrement dit, il confie aux rois des préceptes de droit, les themistes, leur permettant de maintenir l'ordre social au sein de leur communauté s'ils les appliquent convenablement et les font respecter. Pour être porteuses d'ordre, les décisions du roi doivent se fonder sur les themistes dont ils deviennent les interprètes. Employé au pluriel, themistes désigne des préceptes ou prescriptions de différents types, indiquant

[66] Odyssée, XI, 185-187. Sur la précarité du pouvoir réellement assumé par Télémaque, cf. CANTARELLA 2003, p. 120-129.

[67] Sur les traits fondamentaux des basileis homériques, cf. Carlier 1984, p. 141-190.

[68] Iliade, I, 237-239.

[69] Iliade, XVIII, 497-508.

[70] CARLIER 1996, p. 5-22.

[71] Odyssée, XI, 568-571. CANTARelLa 2002, p. 281-283 ; Carlier 2006, p. 101-110 ; LÉVy 2010, p. 339-365.

[72] Hymne homérique à Déméter, 103 et 473 : évoque les rois themistopoloi.

[73] Iliade, IX, 99 et Iliade, II, 206.

[74] RUDHARDT 1999, p. 19-29 et 155-160. la conduite que la themis requiert dans une situation donnée. Elles s'entendent comme l'expression plus concrète de la themis conçue comme une exigence d'ordre ou d'équilibre supposée inspirer I'homme dans ses conduites [74]. Les themistes ont pu être interprétées comme des prescriptions oraculaires, rappelant la fonction mantique de Themis et renforçant le rôle de Zeus comme source d'inspiration du droit [75]. Toutefois, seule une occurrence incline en ce sens. Quand les prétendants ambitionnent d'assassiner Télémaque, Amphinomos préconise avant tout de consulter les dieux : « si nous avons pour nous un arrêt du grand Zeus ( $\Delta$ ıós $\mu \varepsilon \gamma$ ád $\lambda$ ıı

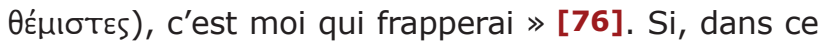
passage, les themistes peuvent être associées à une procédure oraculaire énonçant les conduites que la themis conseille dans un cas singulier, cela n'autorise pas pour autant à généraliser au risque d'occulter les themistes prononcées à l'agora par un roi qui tient le sceptre et se réclame de Zeus. Les themistes constituent plus généralement des prescriptions judiciaires, des règles de comportement ou des principes de gouvernement pouvant servir de fondement aux décisions politiques [77].

Si Zeus a transmis aux rois la connaissance des themistes afin qu'ils puissent administrer la justice, les Muses leur donnent les compétences nécessaires pour énoncer une droite dikê. Le juge juste, dikaios, doit faire preuve de sagesse et d'adresse dans l'élaboration de sa dikê mais surtout de persuasion. Selon Hésiode, les Muses donnent aux rois la compétence de «bien parler», de prononcer un discours persuasif à même de convaincre du bien-fondé de la décision. La dikê apparaît comme un acte de parole performative dont l'efficacité tient à la qualité du discours persuasif employé par le roi. Inspirée par les divinités, la parole du roi acquiert une valeur performative [78].

Les basileis semblent détenir une connaissance privilégiée des themistes, leur permettant d'assumer

[75] HiRZEL 1907, p. 10-17 ; GeRnet 1982 (1968), p. 57, 127, 243 et 1982 ; RuzÉ 1997, p. 97-98; CANTARELLA 2003 , p. $282 n^{\circ} 2$; SCHEID-TISSINIER 2010, p. 26 signale le rôle des divinités et plus particulièrement de Zeus comme garants de l'ordre social, mais rejette toute inspiration divine de la themis ou des themistes.

[76] Odyssée, XVI, 403.

[77] Iliade, I, 238-239 ; II, 204-205 ; IX, 98-99 ; XVI, 387-388; Hésiode, Théogonie, 9-10.

[78] Papakonstantinou 2008, p. 26 et suivantes ; Detienne 2006 (1967), p. 102-105 souligne le lien étroit entre pratiques mantiques, activité politique et fonction judiciaire des basileis : les décisions du roi pouvant s'apparenter à des oracles ; en ce sens, le roi agit comme intermédiaire entre les divinités et le monde des hommes. 
une fonction normative et juridictionnelle déléguée par la divinité. Les basileis sont qualifiés de « nour-

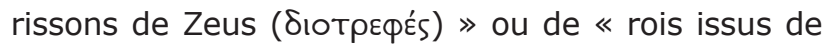
Zeus (ék $\delta \varepsilon \Delta \hat{i} O S) \gg[79]$. En donnant le sceptre et les themistes aux rois, Zeus leur confère la compétence de dicter des sentences en son nom. Tenir ou détenir le sceptre revient à être investi d'un pouvoir dont Zeus est à la fois le garant et le premier représentant. Chargés d'appliquer le droit, les basileis deviennent les dépositaires d'un pouvoir décisionnel symbolisé par le sceptre conférant à son détenteur une autorité légitimée par Zeus [80]. Les basileis sont chargés de préserver ou de rétablir un équilibre social altéré en tenant lieu de médiateur ou de juges pacificateurs au nom de Zeus. En l'absence d'un système répressif institutionnalisé, les basileis doivent maintenir la concorde, mais seules les divinités ou la pression sociale sanctionnent les comportements. Parmi les dieux, Zeus joue un rôle de premier plan dans la sanction des comportements enfreignant les règles en usage dans l'épopée : Zeus est présenté comme «l'arbitre des combats humains » tandis que «la balance de Zeus » constitue une image récurrente dans les poèmes [81]. Le respect des dieux et la crainte qu'ils inspirent influencent directement les comportements des hommes. Ce double sentiment les incite, voire les oblige, à respecter les règles et les usages traditionnellement reconnus par la société homérique [82]. De manière générale, quiconque ne se conduit pas avec dikê est puni par les dieux, non seulement les hommes mais également les rois. L'Odyssée décrit le « roi parfait [comme celui] qui, redoutant les dieux, vit selon la dikê »[83], tandis que dans I'Iliade, le mépris de la dikê suscite la colère de Zeus qui « déverse l'eau du ciel avec le plus de violence pour manifester sa colère aux mortels à qui il en veut, à ceux qui sur l'agora, brutalement prononcent des

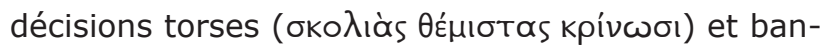

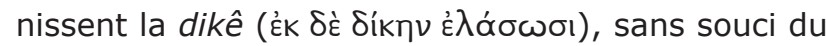
respect dû aux dieux 》 [84].

Dans la conception homérique du droit, divinité et justice sont donc intimement liées. Ne pas respecter la dikê revient à ne pas respecter les dieux, et plus particulièrement à offenser la timé de Zeus, protecteur de la dikê et des themistes. Zeus exerce une justice distributive qui, associée à la conception d'une sanction divine déclenchée par certaines attitudes, exerce manifestement une fonction dissuasive contribuant à garantir le respect du système normatif homérique en encadrant le pouvoir des basileis et en contenant les déviances comportementales [85].

\section{CONCLUSION : CONCEVOIR LE DROIT DE LA HAUTE ÉPOQUE ARCHAïQUE GRECQUE À TRAVERS LA TRANSCRIPTION ÉPIQUE}

Les poèmes décrivent le fonctionnement d'une société de tradition orale représentative des communautés grecques dominées par de puissants seigneurs de guerre entre le $x^{e}$ et le viII siècle avant J.-C. Dans leur transcription épique, les pratiques juridiques, les acteurs juridictionnels et les sources $d$ 'inspiration du droit expriment une conception du droit propre aux communautés grecques de la haute époque archaïque. L'épopée révèle ainsi l'existence d'un droit hiérarchisé dès le haut archaïsme grec.

La société homérique se fonde sur un système normatif et juridictionnel régulé par Zeus qui encadre les conduites des hommes. Il confie aux basileis les principes de bon gouvernement afin qu'ils assument, en son nom, l'autorité juridictionnelle en respectant l'impératif d'équilibre exigé par la themis.

La finalité du droit homérique s'avère moins de punir la violation des règles coutumières que de favoriser la résolution pacifique des conflits. Dans un tel système, la qualité première d'un juge n'est pas de connaître parfaitement les normes générales mais de prouver sa compétence à formuler un arbitrage consenti et approuvé par la communauté. La société homérique dispose de différents procédés pour garantir le respect du droit et préserver I'ordre social. Certains interviennent pour prévenir I'infraction aux normes, tandis que d'autres interviennent une fois l'infraction commise et l'équilibre altéré. Malgré l'absence d'un système répressif institutionnalisé, la société homérique est donc à même de guider et de contrôler les comportements humains grâce à un droit hiérarchisé.

[79] Notamment Iliade, V, 463 ; XVII, 34 ; XVII, 685. Dans le cas d'Agamemnon, cette filiation s'avère d'autant plus forte qu'il descend effectivement de Zeus, cf. Iliade, II, 101-105.

[80] Sur le sceptre, cf. Carlier 1984, p. 191-192 et BOUVIER 2002, p. 273-275.

[81] Iliade, VIII, 69-72; XIV, $658 ;$ XIX, 221-224 ; XXII, 208-213.

[82] RUDHART 1999, p. 112 parle de «dikè subjective » qu'il oppose à la « dikè objective ».

[83] Odyssée, XIX, 109-110.

[84] Iliade, XVI, 385-389.

[85] Cf. également Odyssée, XIX, 110-115; Iliade, XVI, 389-393; XXIV, 527 et Hésiode, Travaux et Jours, 220. 
Benveniste, Émile, 1968, Le vocabulaire des institutions indo-européennes, tome II, Paris, p. 99-110.

Bouvier, David, 2002, Le sceptre et la lyre. L'Iliade ou les héros de la mémoire, Grenoble.

Cantarella, Éva, 2002, « Dispute Settlement in Homer: Once Again on the Schield of Achilles' », dans Julia Strangas et alii (éd.), Mélanges en l'honneur de Panayotis D. Dimakis. Droits antiques et société, Athènes, p. 147-165.

Cantarella, Eva, 2003, Ithaque : de la vengeance d'Ulysse à la naissance du droit, Strasbourg.

Du SABlon, Vincent, 2014, Le système conceptuel de l'ordre du monde dans la pensée grecque à l'époque archaïque, Namur.

Carlier, Pierre, 1984, La royauté en Grèce avant Alexandre, Strasbourg.

CARlier, Pierre, 2006, «Anax and basileus in the Homeric poems », dans Sigrid Deger-Jalkotzy \& Irène Lemos (éd.), 2006, Ancient Greece. From the Mycenaean Palaces to the Age of Homer, Edinburgh, p. 101-110.

Corsano, Marinella, 1988, Themis, la norma e l'oracolo nella Grecia antica, Lecce.

Detienne, Marcel, 2001, Les maîtres de vérité dans la Grèce archaïque, 2e éd. (1 $1^{\text {re }}$ éd. 1968), Paris.

Dickie, W. Matthew, 1978, «Dike as a moral term in Homer and Hesiod », Classical Philology 73, p. 91-101.

Gagarin, Mickael, 1973, «Dike in the Works and Days », Classical Philology 68, p. 81-94.

Gagarin, Mickael, 1974, «Dike in Archaic Thougth », Classical Philology 69, p. 186-197.

Gernet, Louis, 1982, Anthropologie de la Grèce antique, $2^{\mathrm{e}}$ éd. (1 $1^{\text {re }}$ éd. 1968), Paris.

Gernet, Louis, 2001, Recherche sur le développement de la pensée morale et juridique en Grèce ancienne, $2^{\mathrm{e}}$ éd. (1 $1^{\text {re }}$ éd. 1917), Paris.

GLotz, Gustave, 1904, La solidarité de la famille dans le droit criminel en Grèce, Paris.

Hirzel Rudolf, 1907, Themis, Dike und Verwandtes: ein Beitrag zur Geschichte der Rechtsidee bei den Griechen, Leipzig.

LÉvy, Édmond, 1995, «Arété, timè, aidôs et némésis : le modèle homérique », Ktèma 20, p. 177-211.

LÉvy, Édmond, 1998, «Dikè chez Homère : entre privé et public », Ktèma 23, p. 71-79.

LÉvy, Édmond, 2010, « Le système politique de I'Iliade », Ktèma 35, p. 339-365.

Lo Schiavo, Aldo, 1997, Themis e la sapienza dell'ordine cosmico, Napoli.

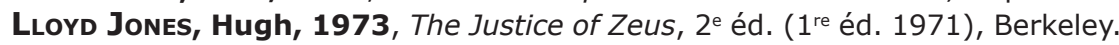

Papakonstantinou, Zinon, 2008, Lawmaking and Adjudication in Archaic Greece, London.

Pelloso, Carlo, 2012, Themis e dike in Omero. Ai primordi del diritto dei Greci, Alessandria.

Pirontr, Gabriella, 2009, «Dans l'entourage de Thémis : les Moires et les «normes» panthéoniques », dans Pierre Brulé, (éd.), La norme en matière religieuse en Grèce ancienne (Kernos supplément 21), Liège, p. 13-27.

RudhARDt, Jean, 1999, Thémis et les Hôrai : Recherche sur les divinités grecques de la justice et de la paix, Genève.

Ruzé, Françoise, 1997, Délibération et pouvoir dans la cité grecque de Nestor à Socrate, Paris.

SCheid-Tissinier, Évelyne, 1994, « À propos du rôle et de la fonction de l'histôr », Revue de philologie, de littérature et $d^{\prime}$ histoire ancienne 68, p. 187-208.

SCHeid-Tissinier, Évelyne, 2008, « Jean Rudhardt et la dikè », Kernos 21, p. 173-184.

Scheid-Tissinier, Évelyne, 2010, «L'énoncé des normes dans le monde homérique et leur application », Métis 8 , p. 21-38. 